\title{
Original
}

\section{Quantitative Structure-Activity Relationship of Antibacterial Dodecylpyridinium lodide Derivatives}

\author{
KIYO OKAZAKI ${ }^{*}$, YUKI MANABE ${ }^{2}$, TAKUYA MAEDA ${ }^{3}$, HIDEAKI NAGAMUNE $^{3}$, \\ AND HIROKI KOURAI ${ }^{3}$

\begin{abstract}
'Faculty of Human Life Science, Shikoku University, Furukawa, Ojin-cho Tokushima 771-11, '2Department of Chemical Science and Technology, and ${ }^{3}$ Department of Biological Science and Technology, Faculty of Engineering, The University of Tokushima, Minamijosanjima-cho, Tokushima 770, Japan
\end{abstract}

Received 25 April 1996/Accepted 5 August 1996

\begin{abstract}
$N$-Dodecylpyridinium iodide derivatives having hydroxyl, amino, methyl, chloro and/or trifluoromethyl groups on the pyridine ring were synthesized from the corresponding pyridines and dodecyl iodide under $80 \mathrm{MPa}$ in order to delineate a quantitative structure-activity relationship. The bactericidal and bacterioclastic activity of the derivatives against Escherichia coli K12 W3110 was strongly affected by the kind of substituent groups these derivatives possesed and their positions. The electron-releasing groups such as amino and methyl groups markedly enhanced such activity, while the electron-attracting groups such as carboxyl and carbamoyl groups reduced it. The bactericidal activity of derivatives was dependent on acidic dissociation constant of the corresponding pyridines and the chemical shift of methylene protons adjacent to the ammonium nitrogen. When bactericidal activity was plotted against bacterioclastic activity, the relationship was found to be linear. There was no correlation, however, between bactericidal activity and hydrophobicity of derivatives. In conclusion, our findings suggest that the bactericidal activity of $\mathbf{N}$-dodecylpyridinium derivatives is dependent on the electron density of the ammonium nitrogen, and also on the bacterioclastic activity.
\end{abstract}

Key words: Biocides/Pyridinium salts/Bactericidal activity/Bacterioclastic activity/Quantitative structure-activity relationship.

\section{INTRODUCTION}

Quaternary ammonium compounds (QAC) have been used widely in food industries, textile industries and hospitals as disinfectants. Some of them have potent bactericidal and fungicidal activity. Krysinski (1990), Csiba et al. (1987), Kourai et al. (1970; 1972; 1973; 1985a; 1986b; 1989; 1994b) and Yabuhara et al. (1972) reported on the antimicrobial characteristics of various series of QAC against bacteria, yeasts, and fungi. The antimicrobial activity was affected by the alkyl chain length (Devinsky et al., 1990; Kourai et al., 1978; 1980a), the adsorbability

"Corresponding author. Tel: +81-886-65-1300, Fax : + 81-886-65-8037.
(Kourai et al., 1980b; 1983a) and the hydrophobicity (Kourai et al., 1983b; 1983c). The activity of pyridinium salts (Krysinski, 1991; Kourai et al., 1985b; 1986a; 1994c) was also significantly affected by chemical structure of the ammonium moiety. In addition, Kourai et al. (1985b) reported that there was a relationship between the antimicrobial activity of pyridinium derivatives and the acidic dissociation constant ( $\mathrm{pKa}$ ) for the corresponding pyridines. We further reported on the relationship between the antimicrobial and bacterioclastic activity of other QAC (Kourai et al., 1994a; 1994c; 1995).

In this study, $\mathrm{N}$-dodecylpyridinium iodide derivatives (12 compounds), having various substituents on the pyridine ring, were synthesized in order to delineate a quantitative relationship between its structure 
and antibacterial activity. The bactericidal and bacterioclastic activity of the new compounds against Escherichia coli K12 W3110 was measured together with 17 compounds of QAC which were previously synthesized in our laboratory (Kourai et al., 1985b). Then the activity was analyzed with their physicochemical parameters such as the acidic dissociation constant (pKa), the chemical shift ( $\delta \mathrm{ppm}$ ) of ${ }^{1} \mathrm{H}$ NMR and their hydrophobicity $\left(R_{M}\right)$.

\section{MATERIALS AND METHODS}

\section{Chemicals}

All chemicals for the synthesis of $N$-dodecylpyridinium derivatives were reagent grade commercial materials and used without further purification. Table 1 shows the chemical structures and abbreviations of the tested dodecylpyridinium derivatives.

\section{Synthesis of $\boldsymbol{N}$-dodecylpyridinium derivatives}

The derivatives were prepared from corresponding pyridines: 3-hydroxypyridine, 2,3-diaminopyridine, 3,4-diaminopyridine, 2-amino-3-hydroxypyridine, 2amino-4-methylpyridine, 2-amino-5-methylpyridine, 3hydroxy-6-methylpyridine, 2-amino-5-chloropyridine, 2-amino-3,5-dichloropyridine, 2-amino-5-trifluoromethylpyridine, 3-(1-hydroxyethyl) pyridine, and 2-(2hydroxyethyl) pyridine. A mixture of $0.1 \mathrm{~mol}$ of each pyridine and $0.1 \mathrm{~mol}$ of dodecyl iodide in $50 \mathrm{ml}$ of absolute ethyl alcohol was heated at $80^{\circ} \mathrm{C}$ for $18 \mathrm{~h}$ under $80 \mathrm{MPa}$ of static pressure with a YHP-92 high pressure reactor. After ethyl alcohol in the reaction mixture was removed with a rotary evaporator under a reduced pressure, the residue was recrystallized from ethyl alcohol-diethyl ether (1:10) to give an $\mathrm{N}$ dodecylpyridinium derivative as white prisms.

\section{Nuclear Magnetic Resonance Spectroscopy}

All the ${ }^{1} \mathrm{H}-\mathrm{NMR}$ experiments were performed with a JEOL JEM-EX400 spectrometer at $400 \mathrm{MHz}$. Tetramethylsilane was used as an internal standard, and the chemical shift was presented as $\delta$ value.

\section{Minimum bactericidal concentration (MBC)}

E. coli W3110 was used for the measurement of $\mathrm{MBC}$ of $\mathrm{N}$-dodecylpyridinium derivatives. The bacterium was cultured in $20 \mathrm{ml}$ of nutrient broth (Difco) with shaking for $18 \mathrm{~h}$ at $37^{\circ} \mathrm{C}$. The bacterial culture $(1$ $\mathrm{ml}$ ) was inoculated into $100 \mathrm{ml}$ of nutrient broth, which was incubated for $2 \mathrm{~h}$ at $37^{\circ} \mathrm{C}$. The exponentially growing cells in the incubated broth were harvested by centrifugation at $5,000 \times \mathrm{g}$ for $10 \mathrm{~min}$ at $2^{\circ} \mathrm{C}$, washed and suspended in sterilized ice-cooled water. The cell concentration of the suspension was adjusted to $4 \times 10^{6}$ cells per $\mathrm{ml}$ with ice-cooled water. One $\mathrm{ml}$ of an aqueous solution containing $1 \mathrm{mg}$ of a derivative was diluted stepwise with sterilized water. After each $0.5 \mathrm{ml}$ portion of the diluted solution was mixed with $0.5 \mathrm{ml}$ of the cell suspension, the mixtures were incubated in a water bath shaker for $30 \mathrm{~min}$ at $37^{\circ} \mathrm{C}$. Then $0.1 \mathrm{ml}$ portions of mixtures were taken out and inoculated into $2 \mathrm{ml}$ of nutrient broth containing $1 \%(\mathrm{v} / \mathrm{v})$ polyoxyethylene sorbitan monooleate (Tween 80, Nakarai Tesque, Inc.). After the inoculated broth was incubated at $37^{\circ} \mathrm{C}$ for $24 \mathrm{~h}, \mathrm{MBC}$ was determined by visual inspection.

\section{Critical vesiculation concentration (CVC)}

CVC of the dodecylpyridinium derivative against exponential phase cells of $E$. coli W3110 was measured in terms of the increase in turbidity of the cell suspensions. Three $\mathrm{ml}$ of the cell suspension, which was kept in an ice bath, was preincubated at $37^{\circ} \mathrm{C}$ for 2 min. After prescribed amounts of aqueous solution of a derivative was added to the suspension, the optical density of the suspension at $660 \mathrm{~nm}\left(\mathrm{OD}_{660}\right)$ was measured continuously at $37^{\circ} \mathrm{C}$ for $200 \mathrm{~s}$ with a Shimadzu UV-160 spectrophotometer equipped with a TCC-240A, a thermo-electrically temperaturecontrolled cell holder. A plot of the turbidity increments $\left(\triangle \mathrm{OD}_{660} / 200 \mathrm{~s}\right)$ of the cell suspension vs. the concentration of the added derivative gave two straight lines. The concentration determined from the point of intersection of the two lines was defined as CVC (Kourai et al., 1994a).

\section{Acidic dissociation constant (pKa) of pyridines}

The pKa of the corresponding pyridine was measured by a pH-titration method (Herbert et al., 1956) with $0.01 \mathrm{~N}$ hydrochloric acid at $25^{\circ} \mathrm{C}$. The pKa data of other pyridines were quoted from our previous paper (Kourai et al., 1985b).

\section{Hydrophobicity parameters of the pyridinium de- rivative}

The $R_{M}$ value, which can be determined very easily by partition chromatography with reversed phases was used as a hydrophobicity parameter (Kourai et al., 1994c; 1995). The pyridinium derivative was applied to a reversed phase thin-layer chromatography plate (DC-Fertigplatten, RP-18 $\mathrm{F}_{254} \mathrm{~S}$, Merck Co.) and chromatographed with an acetonitrile-ethyl alcoholwater (10:9:1) solvent system at $20^{\circ} \mathrm{C}$. The flow rate of samples was determined under irradiation with UV light. The $R_{M}$ value was calculated as follows:

$$
R_{M}=\log \left(1 / R_{f}-1\right)
$$

where $R_{f}$ is the flow rate of the pyridinium derivative sample. 
TABLE 1. Chemical structures of dodecylpyridinium iodide derivatives.

\begin{tabular}{|c|c|c|c|c|c|c|}
\hline \multirow{2}{*}{ No. } & \multirow{2}{*}{ Abbrev. } & \multicolumn{5}{|c|}{ Substituent groups } \\
\hline & & 2 & 3 & 4 & 5 & 6 \\
\hline 1 & $P$ & $\mathrm{H}$ & $\mathrm{H}$ & $\mathrm{H}$ & $\mathrm{H}$ & $H$ \\
\hline 2 & $2 \mathrm{M}$ & $\mathrm{CH}_{3}$ & $\mathrm{H}$ & $\mathrm{H}$ & $\mathrm{H}$ & $\mathrm{H}$ \\
\hline 3 & $3 M$ & $\mathrm{H}$ & $\mathrm{CH}_{3}$ & $H$ & $\mathrm{H}$ & $\mathrm{H}$ \\
\hline 4 & $4 \mathrm{M}$ & $\mathrm{H}$ & $\mathrm{H}$ & $\mathrm{CH}_{3}$ & $\mathrm{H}$ & $\mathrm{H}$ \\
\hline 5 & $24 \mathrm{DM}$ & $\mathrm{CH}_{3}$ & $\mathrm{H}$ & $\mathrm{CH}_{3}$ & $\mathrm{H}$ & $\mathrm{H}$ \\
\hline 6 & $26 \mathrm{DM}$ & $\mathrm{CH}_{3}$ & $\mathrm{H}$ & $\mathrm{H}$ & $\mathrm{H}$ & $\mathrm{CH}_{3}$ \\
\hline 7 & $34 \mathrm{DM}$ & $\mathrm{H}$ & $\mathrm{CH}_{3}$ & $\mathrm{CH}_{3}$ & $\mathrm{H}$ & $\mathrm{H}$ \\
\hline 8 & $35 \mathrm{DM}$ & $\mathrm{H}$ & $\mathrm{CH}_{3}$ & $\mathrm{H}$ & $\mathrm{CH}_{3}$ & $\mathrm{H}$ \\
\hline 9 & 246TM & $\mathrm{CH}_{3}$ & $\mathrm{H}$ & $\mathrm{CH}_{3}$ & $\mathrm{H}$ & $\mathrm{CH}_{3}$ \\
\hline 10 & 3CABA & $\mathrm{H}$ & $\mathrm{CONH}_{2}$ & $\mathrm{H}$ & $\mathrm{H}$ & $\mathrm{H}$ \\
\hline 11 & $4 \mathrm{CABA}$ & $\mathrm{H}$ & $\mathrm{H}$ & $\mathrm{CONH}_{2}$ & $\mathrm{H}$ & $\mathrm{H}$ \\
\hline 12 & 2CABO & $\mathrm{COOH}$ & $\mathrm{H}$ & $\mathrm{H}$ & $\mathrm{H}$ & $\mathrm{H}$ \\
\hline 13 & $3 \mathrm{CABO}$ & $\mathrm{H}$ & $\mathrm{COOH}$ & $\mathrm{H}$ & $\mathrm{H}$ & $\mathrm{H}$ \\
\hline 14 & 4CABO & $\mathrm{H}$ & $\mathrm{H}$ & $\mathrm{COOH}$ & $H$ & $H$ \\
\hline 15 & $2 \mathrm{~A}$ & $\mathrm{NH}_{2}$ & $\mathrm{H}$ & $\mathrm{H}$ & $\mathrm{H}$ & $\mathrm{H}$ \\
\hline 16 & $3 A$ & $\mathrm{H}$ & $\mathrm{NH}_{2}$ & $\mathrm{H}$ & $\mathrm{H}$ & $\mathrm{H}$ \\
\hline 17 & $4 \mathrm{~A}$ & $\mathrm{H}$ & $\mathrm{H}$ & $\mathrm{NH}_{2}$ & $\mathrm{H}$ & $H$ \\
\hline 18 & $3 \mathrm{OH}$ & $\mathrm{H}$ & $\mathrm{OH}$ & $\mathrm{H}$ & $\mathrm{H}$ & $\mathrm{H}$ \\
\hline 19 & $23 D A$ & $\mathrm{NH}_{2}$ & $\mathrm{NH}_{2}$ & $\mathrm{H}$ & $\mathrm{H}$ & $\mathrm{H}$ \\
\hline 20 & $34 \mathrm{DA}$ & $\mathrm{H}$ & $\mathrm{NH}_{2}$ & $\mathrm{NH}_{2}$ & $\mathrm{H}$ & $\mathrm{H}$ \\
\hline 21 & $2 \mathrm{~A} 3 \mathrm{OH}$ & $\mathrm{NH}_{2}$ & $\mathrm{OH}$ & $\mathrm{H}$ & $\mathrm{H}$ & $\mathrm{H}$ \\
\hline 22 & $2 \mathrm{~A} 4 \mathrm{M}$ & $\mathrm{NH}_{2}$ & $\mathrm{H}$ & $\mathrm{CH}_{3}$ & $H$ & $\mathrm{H}$ \\
\hline 23 & $2 \mathrm{~A} 5 \mathrm{M}$ & $\mathrm{NH}_{2}$ & $\mathrm{H}$ & $\mathrm{H}$ & $\mathrm{CH}_{3}$ & $\mathrm{H}$ \\
\hline 24 & $3 \mathrm{OH} 6 \mathrm{M}$ & $\mathrm{H}$ & $\mathrm{OH}$ & $\mathrm{H}$ & $\mathrm{H}$ & $\mathrm{CH}_{3}$ \\
\hline 25 & $2 \mathrm{~A} 5 \mathrm{C}$ & $\mathrm{NH}_{2}$ & $\mathrm{H}$ & $\mathrm{H}$ & $\mathrm{Cl}$ & $\mathrm{H}$ \\
\hline 26 & 2A35DC & $\mathrm{NH}_{2}$ & $\mathrm{Cl}$ & $\mathrm{H}$ & $\mathrm{Cl}$ & $\mathrm{H}$ \\
\hline 27 & $2 \mathrm{~A} 3 \mathrm{C} 5 \mathrm{TF}$ & $\mathrm{NH}_{2}$ & $\mathrm{Cl}$ & $\mathrm{H}$ & $\mathrm{CF}_{3}$ & $H$ \\
\hline 28 & $31 \mathrm{HE}$ & $\mathrm{H}$ & $\mathrm{CH}(\mathrm{OH}) \mathrm{CH}_{3}$ & $H$ & $\mathrm{H}$ & $H$ \\
\hline 29 & $22 \mathrm{HE}$ & $\mathrm{CH}_{2} \mathrm{CH}_{2} \mathrm{OH}$ & $\mathrm{H}$ & $\mathrm{H}$ & $\mathrm{H}$ & $\mathrm{H}$ \\
\hline
\end{tabular}

1. $\mathrm{N}$-dodecylpyridinium iodide, 2. $\mathrm{N}$-dodecyl-2-methylpyridinium iodide, 3. $\mathrm{N}$-dodecyl-3-methylpyridinium iodide, 4. $\mathrm{N}$-dodecyl-4-methylpyridinium iodide, 5. N-dodecyl-2,4-dimethylpyridinium iodide, 6. N-dodecyl-2,6-dimethylpyridinium iodide, 7. N-dodecyl-3,4-dimethylpyridinium iodide, 8. N-dodecyl-3,5-dimethylpyridinium iodide, 9. N-dodecyl-2,4,6-trimethylpyridinium iodide, 10. $\mathrm{N}$-dodecyl-3-carbamoylpyridinium iodide, 11. N-dodecyl-4-carbamoylpyridinium iodide, 12. $\mathrm{N}$ dodecyl-2-carboxypyridinium iodide, 13. N-dodecyl-3-carboxypyridinium iodide, 14. N-dodecyl4-carboxypyridinium iodide, 15. $\mathrm{N}$-dodecyl-2-aminopyridinium iodide, 16. $\mathrm{N}$-dodecyl-3-aminopyridinium iodide, 17. N-dodecyl-4-aminopyridinium iodide, 18. $\mathrm{N}$-dodecyl-3-hydroxypyridinium iodide, 19. $\mathrm{N}$-dodecyl-2,3-diaminopyridinium iodide, 20. $\mathrm{N}$-dodecyl-3,4-diaminopyridinium iodide, 21. $\mathrm{N}$-dodecyl-2-amino-3-hydroxypyridinium iodide, 22. $\mathrm{N}$-dodecyl-2-amino4-methylpyridinium iodide, 23. N-dodecyl-2-amino-5-methylpyridinium iodide, 24. N-dodecyl-3hydroxy-6-methylpyridinium iodide, 25. N-dodecyl-2-amino-5-chloropyridinium iodide, 26. Ndodecyl-2-amino-3,5-dichloropyridinium iodide, 27. N-dodecyl-2-amino-3-chloro-5-trifluoromethylpyridinium iodide, 28. N-dodecyl-3-(1-hydroxyethyl) pyridinium iodide, 29. N-dodecyl-2(2-hydroxyethyl) pyridinium iodide. 
RESULTS AND DISCUSSION

\section{Chemical properties of synthesized dodecyl- pyridinium derivative}

Table 2 shows the data of elemental analysis, melting points and yields of synthesized dodecylpyridinium derivatives. The data for the elemental analysis are in fair agreement with their theoretical values. The NMR data for derivatives are shown in Table 3. The signals of 4.106-4.651 ppm $(2 \mathrm{H}, \mathrm{t})$ and $0.892-0.895 \mathrm{ppm}(3 \mathrm{H}, \mathrm{t})$ were assigned to the methylene proton of $\mathrm{N}^{+}-\mathrm{CH}_{2}$ - and terminal methyl proton of $-\mathrm{CH}_{3}$, respectively. The signals of 1.280-1.290 $\operatorname{ppm}(16 \mathrm{H}, \mathrm{m}), 1.317-1.445 \mathrm{ppm}(2 \mathrm{H}, \mathrm{m})$ and 1.794-

TABLE 2. Chemical properties of dodecylpyridinium iodide derivatives.

\begin{tabular}{|c|c|c|c|c|c|c|c|c|c|}
\hline \multirow{3}{*}{ No. } & \multirow{3}{*}{$\begin{array}{l}\text { lodide } \\
\text { (abbrev.) }\end{array}$} & \multicolumn{6}{|c|}{ Elemental analysis } & \multirow{3}{*}{$\begin{array}{l}\text { m.p. } \\
\left({ }^{\circ} \mathrm{C}\right)\end{array}$} & \multirow{3}{*}{$\begin{array}{l}\text { Yield } \\
\text { (\%) }\end{array}$} \\
\hline & & \multicolumn{2}{|c|}{$C$} & \multicolumn{2}{|c|}{$\mathrm{H}$} & \multicolumn{2}{|c|}{$\mathrm{N}$} & & \\
\hline & & Found & Calc. & Found & Calc. & Found & Calc. & & \\
\hline 18 & $3 \mathrm{OH}$ & 52.17 & 52.18 & 7.61 & 7.73 & 3.41 & 3.58 & $79-80$ & 25.6 \\
\hline 19 & 23DA & 50.30 & 50.37 & 7.61 & 7.95 & 10.22 & 10.36 & $81-83$ & 68.9 \\
\hline 20 & 34DA & 50.42 & 50.37 & 7.88 & 7.95 & 10.21 & 10.36 & $98-100$ & 82.2 \\
\hline 21 & $2 \mathrm{~A} 3 \mathrm{OH}$ & 50.08 & 50.25 & 7.68 & 7.68 & 6.89 & 6.89 & $141-143$ & 87.4 \\
\hline 22 & $2 \mathrm{~A} 4 \mathrm{M}$ & 53.46 & 53.46 & 8.01 & 8.23 & 6.71 & 6.93 & $59-60$ & 85.6 \\
\hline 23 & $2 \mathrm{~A} 5 \mathrm{M}$ & 53.25 & 53.46 & 8.22 & 8.23 & 6.80 & 6.93 & $120-123$ & 80.2 \\
\hline 24 & $30 \mathrm{H} 6 \mathrm{M}$ & 53.19 & 53.33 & 7.72 & 7.96 & 3.52 & 3.46 & $138-140$ & 66.9 \\
\hline 25 & $2 \mathrm{~A} 5 \mathrm{C}$ & 48.07 & 48.07 & 6.99 & 7.12 & 6.50 & 6.59 & $109-111$ & 68.2 \\
\hline 26 & $2 A 35 D C$ & 44.28 & 44.46 & 6.10 & 6.36 & 5.93 & 6.10 & $52-55$ & 67.5 \\
\hline 27 & $2 \mathrm{~A} 3 \mathrm{C} 5 \mathrm{TF}$ & 43.84 & 43.87 & 6.01 & 5.93 & 5.26 & 5.68 & $59-60$ & 55.6 \\
\hline 28 & $31 \mathrm{HE}$ & 54.30 & 54.41 & 8.07 & 8.17 & 3.45 & 3.34 & $44-46$ & 65.3 \\
\hline 29 & $22 \mathrm{HE}$ & 54.63 & 54.41 & 7.91 & 8.17 & 3.29 & 3.34 & $170-172$ & 67.1 \\
\hline
\end{tabular}

TABLE 3. 'H-NMR data of dodecylpyridinium iodide derivatives in $\mathrm{CD}_{3} \mathrm{OD}$.

\begin{tabular}{cll}
\hline No. & $\begin{array}{l}\text { lodide } \\
\text { (abbrev. })\end{array}$ & \multicolumn{1}{c}{ Chemical shift $(\delta \mathrm{ppm})$} \\
\hline 18 & $3 \mathrm{OH}$ & $0.893(3 \mathrm{H}, \mathrm{t}), 1.284(16 \mathrm{H}, \mathrm{m}), 1.377(2 \mathrm{H}, \mathrm{m}), 1.995(2 \mathrm{H}, \mathrm{m}), 4.534(2 \mathrm{H}, \mathrm{t})$, \\
& & $7.855(2 \mathrm{H}, \mathrm{d}), 8.386-8.409(2 \mathrm{H}, \mathrm{m})$ \\
19 & $23 \mathrm{DA}$ & $0.893(3 \mathrm{H}, \mathrm{t}), 1.282(16 \mathrm{H}, \mathrm{m}), 1.373(2 \mathrm{H}, \mathrm{m}), 1.824(2 \mathrm{H}, \mathrm{m}), 4.216(2 \mathrm{H}, \mathrm{t})$, \\
& & $6.760-7.397(3 \mathrm{H}, \mathrm{m})$ \\
20 & $34 \mathrm{DA}$ & $0.893(3 \mathrm{H}, \mathrm{t}), 1.280(16 \mathrm{H}, \mathrm{m}), 1.317(2 \mathrm{H}, \mathrm{m}), 1.861(2 \mathrm{H}, \mathrm{m}), 4.106(2 \mathrm{H}, \mathrm{t})$, \\
& & $6.766(1 \mathrm{H}, \mathrm{d}), 7.650(1 \mathrm{H}, \mathrm{d}), 7.736(1 \mathrm{H}, \mathrm{dd})$ \\
21 & $2 \mathrm{~A} 3 \mathrm{OH}$ & $0.892(3 \mathrm{H}, \mathrm{t}), 1.281(16 \mathrm{H}, \mathrm{m}), 1.374(2 \mathrm{H}, \mathrm{m}), 1.821(2 \mathrm{H}, \mathrm{m}), 4.210(2 \mathrm{H}, \mathrm{t})$, \\
& & $6.775(1 \mathrm{H}, \mathrm{t}), 7.153(1 \mathrm{H}, \mathrm{d}), 7.496(1 \mathrm{H}, \mathrm{d})$ \\
22 & $2 \mathrm{~A} 4 \mathrm{M}$ & $0.892(3 \mathrm{H}, \mathrm{t}), 1.282(16 \mathrm{H}, \mathrm{m}), 1.369(2 \mathrm{H}, \mathrm{m}), 1.794(2 \mathrm{H}, \mathrm{m}), 2.383(3 \mathrm{H}, \mathrm{s})$, \\
& & $4.144(2 \mathrm{H}, \mathrm{t}), 6.786(1 \mathrm{H}, \mathrm{dd}), 6.886(1 \mathrm{H}, \mathrm{s}), 7.862(1 \mathrm{H}, \mathrm{d})$ \\
23 & $2 \mathrm{~A} 5 \mathrm{M}$ & $0.894(3 \mathrm{H}, \mathrm{t}), 1.286(16 \mathrm{H}, \mathrm{m}), 1.379(2 \mathrm{H}, \mathrm{m}), 1.803(2 \mathrm{H}, \mathrm{m}), 2.249(3 \mathrm{H}, \mathrm{s})$, \\
& & $4.137(2 \mathrm{H}, \mathrm{t}), 7.017(1 \mathrm{H}, \mathrm{d}), 7.746(1 \mathrm{H}, \mathrm{dd}), 7.831(1 \mathrm{H}, \mathrm{s})$ \\
24 & $3 \mathrm{OH} 6 \mathrm{M}$ & $0.894(3 \mathrm{H}, \mathrm{t}), 1.290(16 \mathrm{H}, \mathrm{m}), 1.439(2 \mathrm{H}, \mathrm{m}), 1.926(2 \mathrm{H}, \mathrm{m}), 2.741(3 \mathrm{H}, \mathrm{s})$, \\
& & $4.489(2 \mathrm{H}, \mathrm{t}), 7.745(1 \mathrm{H}, \mathrm{d}), 7.792(1 \mathrm{H}, \mathrm{dd}), 8.341(1 \mathrm{H}, \mathrm{d})$ \\
25 & $2 \mathrm{~A} 5 \mathrm{C}$ & $0.895(3 \mathrm{H}, \mathrm{t}), 1.290(16 \mathrm{H}, \mathrm{m}), 1.384(2 \mathrm{H}, \mathrm{m}), 1.811(2 \mathrm{H}, \mathrm{m}), 4.151(2 \mathrm{H}, \mathrm{t})$, \\
& & $7.092(1 \mathrm{H}, \mathrm{d}), 7.890(1 \mathrm{H}, \mathrm{dd}), 8.241(1 \mathrm{H}, \mathrm{d})$ \\
26 & $2 \mathrm{~A} 35 \mathrm{DC}$ & $0.894(3 \mathrm{H}, \mathrm{t}), 1.288(16 \mathrm{H}, \mathrm{m}), 1.398(2 \mathrm{H}, \mathrm{m}), 1.824(2 \mathrm{H}, \mathrm{m}), 4.279(2 \mathrm{H}, \mathrm{t})$, \\
& & $8.272(1 \mathrm{H}, \mathrm{d}), 8.355(1 \mathrm{H}, \mathrm{d})$ \\
27 & $2 \mathrm{~A} 3 \mathrm{C} 5 \mathrm{TF}$ & $0.894(3 \mathrm{H}, \mathrm{t}), 1.288(16 \mathrm{H}, \mathrm{m}), 1.405(2 \mathrm{H}, \mathrm{m}), 1.853(2 \mathrm{H}, \mathrm{m}), 4.337(2 \mathrm{H}, \mathrm{t})$, \\
& & $8.408(1 \mathrm{H}, \mathrm{d}), 8.639(1 \mathrm{H}, \mathrm{d})$ \\
28 & $31 \mathrm{HE}$ & $0.893(3 \mathrm{H}, \mathrm{t}), 1.284(16 \mathrm{H}, \mathrm{m}), 1.389(2 \mathrm{H}, \mathrm{m}), 1.541 \mathrm{and} 1.586(3 \mathrm{H}, \mathrm{dd})$, \\
& & $2.026(2 \mathrm{H}, \mathrm{m}), 4.651(2 \mathrm{H}, \mathrm{t}), 5.093(1 \mathrm{H}, \mathrm{q}), 8.071(1 \mathrm{H}, \mathrm{t}), 8.582(1 \mathrm{H}, \mathrm{d})$, \\
& & $8.902(1 \mathrm{H}, \mathrm{d}), 9.022(1 \mathrm{H}, \mathrm{s})$ \\
29 & $22 \mathrm{HE}$ & $0.893(3 \mathrm{H}, \mathrm{t}), 1.288(16 \mathrm{H}, \mathrm{m}), 1.445(2 \mathrm{H}, \mathrm{m}), 1.984(2 \mathrm{H}, \mathrm{m}), 2.513(2 \mathrm{H}, \mathrm{t})$, \\
& & $3.485(2 \mathrm{H}, \mathrm{t}), 4.617(2 \mathrm{H}, \mathrm{t}), 7.544(1 \mathrm{H}, \mathrm{t}), 7.688(1 \mathrm{H}, \mathrm{dd}), 8.053(1 \mathrm{H}, \mathrm{t})$, \\
& & $8.849(1 \mathrm{H}, \mathrm{dd})$
\end{tabular}


$2.026 \mathrm{ppm}(2 \mathrm{H}, \mathrm{m})$ were assigned to the methylene proton of $-\left(\mathrm{CH}_{2}\right)_{10}$-. These NMR data are consistent with the proposed structures of dodecylpyridinium derivatives. Reversed phase thin-layer chromatograms and high performance liquid chromatograms of the dodecylpyridinium derivatives also showed them to be in a high state of purity (data not shown).

\section{Bactericidal and bacterioclastic activity of dode- cylpyridinium derivatives and their physico- chemical parameters (pKa, $\delta$ and $\boldsymbol{R}_{M}$ )}

The MBC and CVC of dodecylpyridinium iodide derivatives are shown in Table 4. The $\log \mathrm{MBC}^{-1}$ and the $\log \mathrm{CVC}^{-1}$ were defined as their bactericidal activity and bacterioclastic activity, respectively. We have already reported that the introduction of electronreleasing groups, such as amino, methyl and propyl groups, at the 2,4 and/or 6-positions of the pyridine ring enhanced their antimicrobial activity. In this study, almost all iodides having an electron-releasing group on the pyridine ring exhibited strong bactericidal activity. Particularly the iodides having one or two amino substituents on the pyridine ring exhibited strong activity compared with $\mathrm{N}$-dodecylpyridinium iodide $(\mathrm{P})$. The activity was markedly enhanced by introducing two amino groups (no. 19, 20). On the other hand, the activity of iodides (no.10-14) was highly reduced by the introduction of electron-attracting groups such as the carbamoyl and carboxyl groups. Similarly the kind of substituents on the pyridine ring affected their bacterioclastic activity. The introduction of an electron-releasing substituent enhanced their bacterioclastic activity. The acidic dissociation constant ( $\mathrm{pKa}$ ) of the corresponding pyridine, the chemical

TABLE 4. Minimum bactericidal concentrations $(M B C)$ and the critical vesiculation concentrations (CVC) of dodecylpyridinium iodide derivatives, acidic dissociation constants (pKa) of corresponding pyridines, 'H-NMR data of methylene protons adjacent to the ammonium nitrogen of derivatives, and hydrophobicities.

\begin{tabular}{|c|c|c|c|c|c|c|}
\hline No. & $\begin{array}{l}\text { Pyridinium } \\
\text { iodide } \\
\text { (Abbrev.) }\end{array}$ & $\begin{array}{l}\mathrm{MBC} \\
(\mu \mathrm{M})\end{array}$ & $\begin{array}{l}\text { CVC } \\
(\mu \mathrm{M})\end{array}$ & $\mathrm{pKa}^{a}$ & $\begin{array}{l}\delta(\mathrm{ppm}) \\
\mathrm{N}^{+}-\mathrm{CH}_{2^{-}}\end{array}$ & $\begin{array}{l}\text { Hydrophobicity } \\
\left(R_{M}\right)\end{array}$ \\
\hline 1 & $P$ & 71 & 26.9 & 5.19 & 4.677 & -0.047 \\
\hline 2 & $2 M$ & 112 & 26.3 & 5.97 & 4.603 & -0.061 \\
\hline 3 & $3 M$ & 112 & 30.2 & 5.68 & 4.603 & -0.130 \\
\hline 4 & $4 \mathrm{M}$ & 72 & 18.6 & 6.02 & 4.597 & -0.185 \\
\hline 5 & 24DM & 69 & 30.2 & 6.07 & 4.523 & -0.163 \\
\hline 6 & $26 \mathrm{DM}$ & 87 & 12.3 & 6.75 & 4.517 & -0.085 \\
\hline 7 & $34 \mathrm{DM}$ & 69 & 22.9 & 6.34 & 4.526 & -0.178 \\
\hline 8 & $35 \mathrm{DM}$ & 69 & 28.8 & 5.88 & 4.537 & -0.203 \\
\hline 9 & 246TM & 67 & 6.0 & 9.57 & 4.445 & -0.226 \\
\hline 10 & 3CABA & 105 & 31.6 & 3.28 & 4.721 & -0.290 \\
\hline 11 & 4CABA & 204 & 56.2 & 3.52 & 4.705 & -0.341 \\
\hline 12 & $2 \mathrm{CABO}$ & 631 & 87.1 & 1.01 & 4.667 & -0.169 \\
\hline 13 & $3 \mathrm{CABO}$ & 398 & 28.8 & 2.07 & 4.741 & -0.059 \\
\hline 14 & $4 C A B O$ & 802 & 108.0 & 1.84 & 4.661 & -0.169 \\
\hline 15 & $2 \mathrm{~A}$ & 72 & 13.5 & 5.23 & 4.183 & -0.395 \\
\hline 16 & $3 A$ & 46 & 6.0 & 6.86 & 4.437 & -0.321 \\
\hline 17 & $4 \mathrm{~A}$ & 40 & 1.95 & 9.17 & 4.155 & -0.325 \\
\hline 18 & $3 \mathrm{OH}$ & 155 & 39.8 & 4.69 & 4.534 & -0.214 \\
\hline 19 & $23 D A$ & 16 & 6.7 & 6.72 & 4.216 & -0.242 \\
\hline 20 & $34 \mathrm{DA}$ & 10 & 4.3 & 8.90 & 4.106 & -0.261 \\
\hline 21 & $2 \mathrm{~A} 3 \mathrm{OH}$ & 63 & 27.5 & 5.91 & 4.210 & -0.286 \\
\hline 22 & $2 \mathrm{~A} 4 \mathrm{M}$ & 25 & 16.8 & 7.13 & 4.144 & -0.194 \\
\hline 23 & $2 \mathrm{~A} 5 \mathrm{M}$ & 16 & 4.2 & 6.91 & 4.137 & -0.194 \\
\hline 24 & $30 \mathrm{H} 6 \mathrm{M}$ & 63 & 9.5 & 5.35 & 4.489 & -0.227 \\
\hline 25 & $2 A 5 C$ & 25 & 21.5 & 4.61 & 4.151 & -0.233 \\
\hline 26 & 2A35DC & 20 & 24.6 & $N D^{b}$ & 4.279 & -0.244 \\
\hline 27 & 2A3C5TF & 78 & 13.4 & ND & 4.337 & -0.290 \\
\hline 28 & $31 \mathrm{HE}$ & 39 & 21.6 & 4.93 & 4.651 & -0.183 \\
\hline 29 & $22 \mathrm{HE}$ & 39 & 33.9 & 5.24 & 4.617 & -0.106 \\
\hline
\end{tabular}

${ }^{a}$ The values of the pyridines (No.1-17) were quoted from the reference and the other values (No.18-29) were determined in water at $25^{\circ} \mathrm{C}$ by the $\mathrm{pH}$-titration method using $0.01 \mathrm{~N}$ hydrochloric acid.

${ }^{b}$ Undetected 
shift ( $\delta \mathrm{ppm}$ ) of the methylene protons adjacent to ammonium nitrogen of iodides and the hydrophobicity parameter $\left(R_{M}\right)$ of iodides are also summarized in Table 4.

\section{Quantitative structure-activity relationship (QSAR) between bactericidal activity and the acidic dissociation constant}

Figure $1 \mathrm{~A}$ shows the relation between the bactericidal activity $\left(\log \mathrm{MBC}^{-1}\right)$ of iodides and the acidic dissociation constant ( $\mathrm{pKa}$ ) for the corresponding pyridine. The activity against E. coli W3110 increased linearly with an increase in the pKa value. The pKa value of pyridines is directly proportional to the electron density of the nitrogen atom. The $\log \mathrm{MBC}^{-1}$ was analyzed by a QSAR method using pKa at a 95\% confidence level, and an equation was obtained. The correlation coefficient $(r)$ was as high as 0.754 . It was proved that the bactericidal activity of iodides having a dodecyl group against E. coli W3110 is strikingly dependent on the acidic dissociation constant.

\section{Relation between bactericidal activity and the chemical shift}

The electron density of ammonium nitrogen of iodides can also be represented by a chemical shift ( $\delta \mathrm{ppm}$ ) of methylene protons adjacent to ammonium nitrogen. A plot of the $\log \mathrm{MBC}^{-1}$ versus $\delta \mathrm{ppm}$ gave a straight line (Fig. 1B). It suggests that this equation obtained can be regarded as significant by the F-test, and the activity of iodides depends on the chemical shift. Consequently, the bactericidal activity of pyridinium iodides is closely connected with the electron density of the nitrogen atom.

\section{Relation between bactericidal activity and hydro- phobicity}

The bactericidal activity of iodides against E. coli W3110 was plotted against the $R_{M}$ value. As seen in Fig. $1 \mathrm{C}$, there was no correlation between them. It suggests that the $\log \mathrm{MBC}^{-1}$ of iodides with a dodecyl group was scarcely affected by hydrophobicity of iodides. On the other hand, Devinsky et al. (1987) and Pavlikova-Moricka et al. (1994) demonstrated a relationship between lipophilicity (hydrophobicity) and the antimicrobial activity of 4-alkyl-4-ethylmorpholinium bromides and bis-QAC derived from bis-(2dimethylaminoethyl) glutarate which have different alkyl groups, using the QSAR method. In our previous paper (Kourai et al., 1994c), we have also reported that the bactericidal activity of $\mathrm{N}$-alkyl-4-butenylpyridinium bromides (alkyl group; octyl, decyl, dodecyl, tetradecyl, hexadecyl and octadecyl) was correlated with their hydrophobicity. It is thought that the
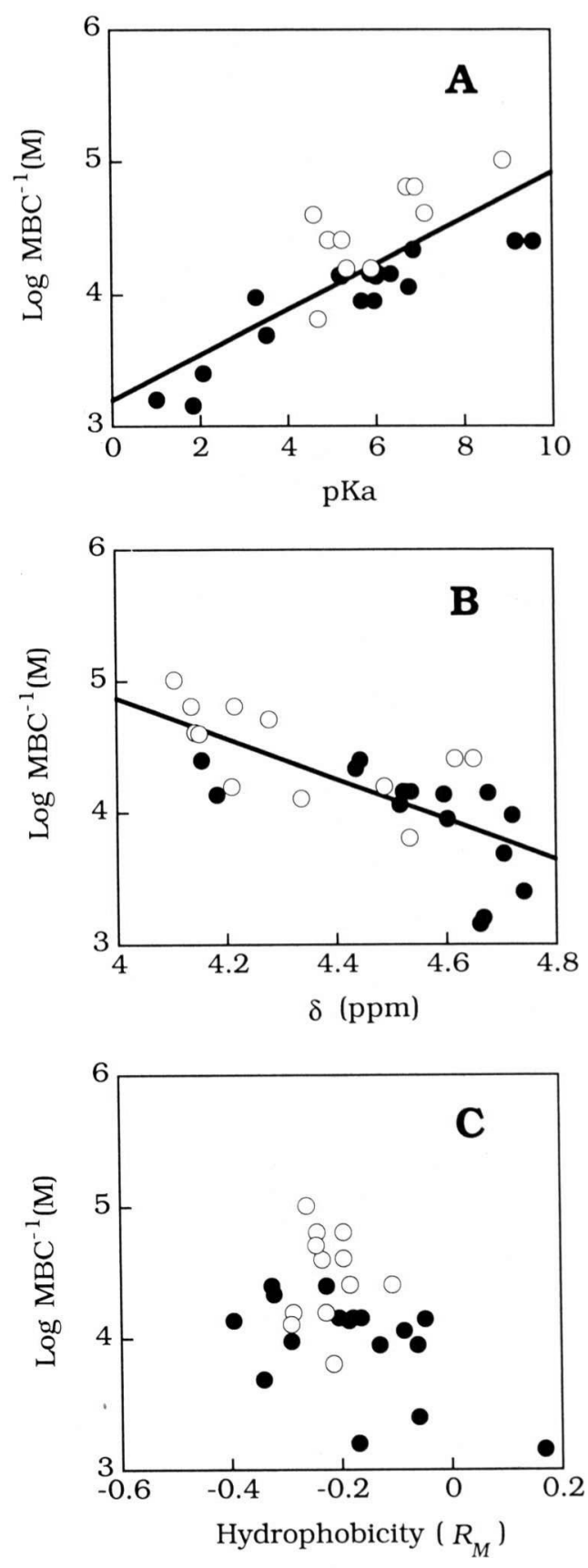

FIG. 1. Relationship between the bactericidal activity (log $\mathrm{MBC}^{-1}$ ) of dodecylpyridinium iodide against exponentially growing cells of $E$. coli W3110 and the acidic dissociation constant ( $\mathrm{pKa}$ ) of the corresponding pyridine $(A)$, the chemical shift ( $\delta \mathrm{ppm}$ ) of the methylene protons adjacent to ammonium nitrogen $(\mathrm{B})$, and the hydrophobicity $\left(R_{M}\right)$ of iodide (C). Symbols: ${ }^{-}$, iodides (no. 1-17); $\bigcirc$, iodides (no. 18-29). Solid lines represent the equations: $(A)$; Log $M B$ $\mathrm{C}^{-1}=0.16( \pm 0.06) \mathrm{pKa}+3.23( \pm 0.35), r=0.754, \quad \mathrm{~F}=32.8$ $\left(F_{0}=4.24\right.$ at $95 \%$ confidence level), (B); $\operatorname{Log~MBC}^{-1}=-1.53$ $( \pm 0.57) \mathrm{ppm}+10.98( \pm 2.52), \quad r=0.730, \quad F=30.8 \quad\left(F_{0}\right.$ $=4.21$ ). 
molecular hydrophobicity of QAC mainly depends on the hydrophobicity of the alkyl group and the pyridinium skeleton of QAC. Therefore it suggests that hydrophobicity correlates with the bactericidal activity among the homologous series of QAC having various lengths of alkyl chains, but the bactericidal activity among various series of pyridinium derivatives having a dodecyl group, used in this study, is not dependent on the $R_{M}$ value.

\section{Relation between bacterioclastic activity and the acidic dissociation constant}

Figure $2 \mathrm{~A}$ shows the relation between the bacterioclastic activity of iodides and the pKa of the corresponding pyridines. Log $\mathrm{CVC}^{-1}$ was linearly correlated to the $\mathrm{pKa}$ value, and the correlation coefficient was 0.828. This coefficient was higher than that $(r$ $=0.754$ ) for the $\log \mathrm{MBC}^{-1}$. This result implies that the $\log \mathrm{CVC}^{-1}$ as well as the $\log \mathrm{MBC}^{-1}$ is dependent on the pKa value.

\section{Relation between bacterioclastic activity and the chemical shift}

Figure $2 \mathrm{~B}$ represents the relation between log $\mathrm{CV}$ $\mathrm{C}^{-1}$ and the chemical shift ( $\delta \mathrm{ppm}$ ) of iodides. The plot gave a straight regression line with 0.663 of the correlation coefficient of the equation. It suggests that bacterioclastic activity is also dependent on chemical shift value.

\section{Relation between bacterioclastic activity and hy- drophobicity}

A plot of bacterioclastic activity against the hydrophobicity of iodides showed no correlation as seen in Fig. 2C. It suggests that bacterioclastic activity, as well as the bactericidal activity of iodides, is scarcely affected by hydrophobicity.

\section{Relation between bactericidal activity and bacte- rioclastic activity}

Figure 3 shows the relation between bactericidal activity and bacterioclastic activity. The $\log \mathrm{MBC}^{-1}$ was observed to increase linearly with the $\log \mathrm{CVC}^{-1}$. The coefficient of the regression equation was 0.700 . It suggests that the bactericidal activity of iodides against E. coli W3110 is extremely dependent on bacterioclastic activity. From this result, the bacterioclastic action of iodide derivatives can be considered to be one stage of the bactericidal activity. The bacterioclastic action is a combined action of blebbing and vesiculation on the bacterial cell surface. The bacterioclastic activity of iodides was influenced by the physicochemical parameters, pKa and chemical shift. These parameters reflect the electron
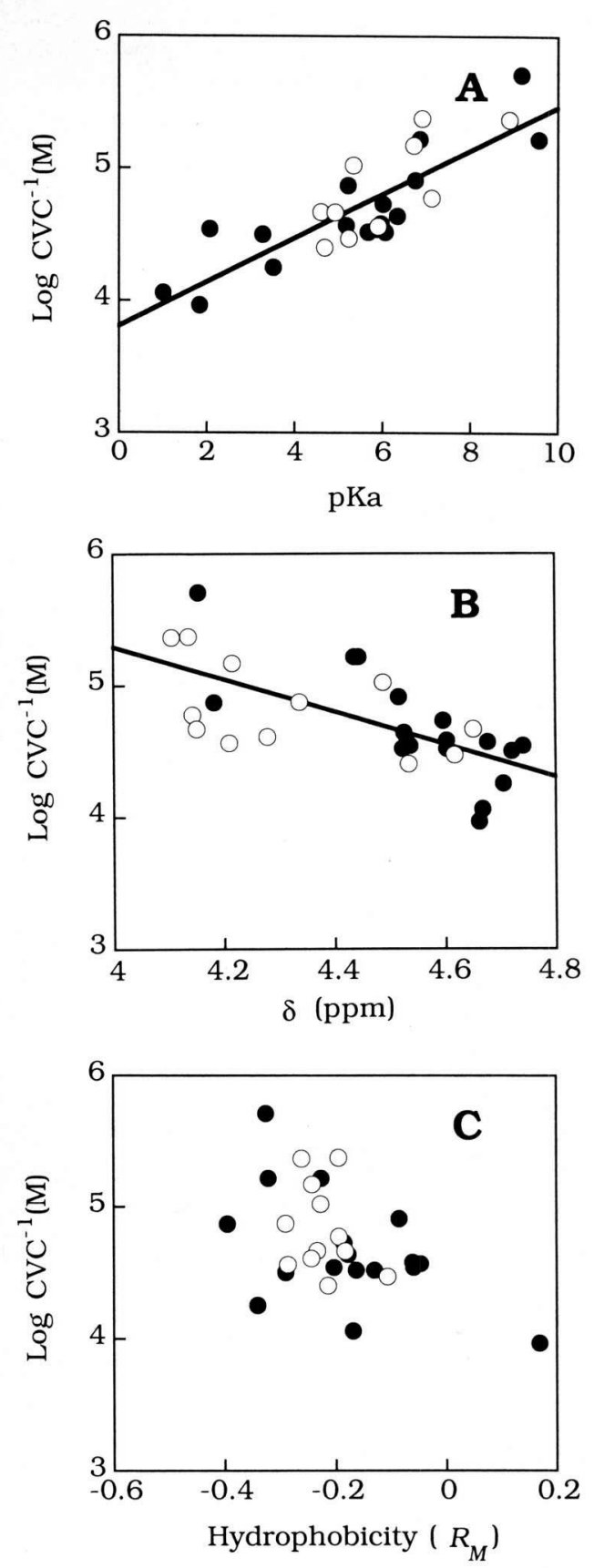

FIG. 2. Relationship between the bacterioclastic activity $\left(\log \mathrm{CVC}^{-1}\right.$ ) of dodecylpyridinium iodide against $E$. coli W3110 and the acidic dissociation constant (pKa) (A), chemical shift ( $\delta \mathrm{ppm})(\mathrm{B})$, and hydrophobicity $\left(R_{M}\right)(\mathrm{C})$. Symbols: $\bigcirc$, iodides (no. 1-17); $\bigcirc$, iodides (no. 18-29). Solid lines represent the equations: $(\mathrm{A})$; $\log \mathrm{CVC}^{-1}=0.17$ $( \pm 0.05) \mathrm{pKa}+3.81( \pm 0.27), r=0.828, F=54.7(\mathrm{Fo}=4.24)$, (B); $\log \mathrm{CVC}^{-1}=-1.24( \pm 0.55) \mathrm{ppm}+10.27( \pm 2.47)$, $r=0.663, F=21.2(F o=4.21)$.

density of the nitrogen atom. It is concluded that the $\mathrm{N}$-dodecylpyridinium iodide derivative which has a high electron density on the nitrogen atom exhibits high bacterioclastic activity and bactericidal activity. 


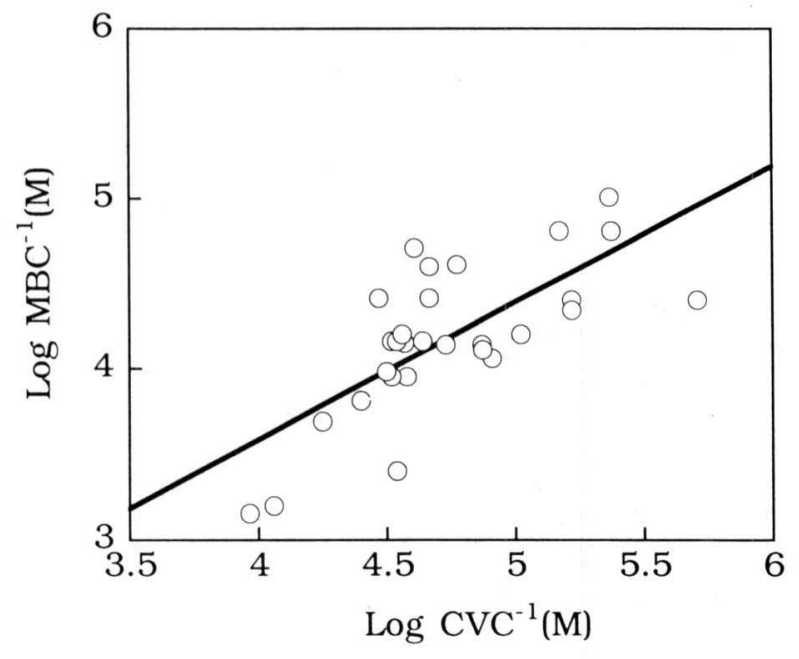

FIG. 3. Relationship between bactericidal activity (log MB $\mathrm{C}^{-1}$ ) and the bacterioclastic activity $\left(\log \mathrm{CVC}^{-1}\right)$ of dodecylpyridinium iodide against $E$. coli W3110. Solid line represents the equation: $\log \mathrm{MBC}^{-1}=0.779( \pm 0.316) \mathrm{Log} \mathrm{CVC}^{-1}$ $+0.479( \pm 1.50), r=0.700, F=25.6\left(F_{0}=4.21\right)$.

\section{REFERENCES}

Brown, H. C., Gintis, D., and Domash, L. (1956) Linear strain energy relationships involving reactants of large steric requirements. J. Am. Chem. Soc., 78, 5387-5394.

Csiba, I., Lacko, I., Bittererova, F., and Mlynarcik, D. (1987) Aggregational properties and antimicrobial activity of $\mathrm{N}$-[(2-alkanoyloxy)ethyl]-dodecyldimethylammonium and $N$-[(2-alkanoylamido) ethyl]dodecyldimethylammonium bromides (in Slovak). Cesk. Farm., 36, 349-354.

Devinsky, F., Lacko, I., Vidlakova, J., and Gallayova, D. (1987) Quantitative relationships between structure, critical micelle concentration, $R_{M}$-values and antimicrobial activity (in Slovak). Cesk. Farm., 36, 141-144.

Devinsky, F., Leitmanova-Kopecka, A., Sersen, F., and Balgavy, P. (1990) Cut-off effect in antimicrobial activity and in membrane perturbation efficiency of the homologous series of $\mathrm{N}, \mathrm{N}$-dimethylalkylamine oxides. J. Pharm. Pharmacol., 42, 790-794.

Kourai, H., and Takeichi, K. (1970) Antimicrobial activity of quaternary ammonium bromide (in Japanese). J. Ferment. Technol., 48, 635-640.

Kourai, H., Takeichi, K., Okazaki, M., Enatsu, T., and Shibasaki, I. (1972) The mode of synergistic action of lauryltrimethyl ammonium bromide on triphenyl lead acetate (in Japanese). J. Ferment. Technol., 50, 34-40.

Kourai, H., Takeichi, K., and Shibasaki, I. (1973) The mode of action of (3-trialkyl tin) propyltrimethyl ammonium iodides (in Japanese). J. Ferment. Technol., 51, 832-839.

Kourai, H., Sato, T., Uchiwa, N., Takeichi, K., and Shibasaki, I. (1978) The antimicrobial characteristics of quaternary ammonium salts (in Japanese). J. Antibact. Antifung. Agents, 6, 11-18.

Kourai, H., Horie, T., Takeichi, K., and Shibasaki, I. (1980a) The antimicrobial characteristics of quaternary ammonium salts and their alkyl chain length (in Japanese). J. Antibact. Antifung. Agents, 8, 191-199.

Kourai, H., Sato, T., Horie, T., Takeichi, K., and Shibasaki, I. (1980b) The antimicrobial characteristics of quaternary ammonium salts (in Japanese). J. Antibact. Antifung. Agents, 8, 233-239.

Kourai, H., Machikawa, F., Horie, T., Takeichi, K., and Shibasaki, I. (1983a) Quantitative relation between antimicrobial activity and adsorbability of $\mathrm{N}$ octylquinolinium iodide on Escherichia coli K12. J. Antibact. Antifung. Agents, 11, 51-54.

Kourai, H., Machikawa, F., Horie, T., Takeichi, K., and Shibasaki, I. (1983b) Quantitative structure-activity correlation on antimicrobial activity and hydrophobicity of quaternary ammonium iodides (in Japanese). J. Antibact. Antifung. Agents, 11, 401408.

Kourai, H., Machikawa, F., Horie, T., Takeichi, K., and Shibasaki, I. (1983c) Quantitative structure-activity correlation on antimicrobial activity and hydrophobicity of $\mathrm{N}$-alkylpyridinium iodide derivatives. J. Antibact. Antifung. Agents, 11, 553-562.

Kourai, H., Takechi, H., Horie, T., Uchiwa, N., Takeichi, K., and Shibasaki, I. (1985a) Antimicrobial characteristics and a mode of action of $\mathrm{N}$-alkylpyridinium iodides against Escherichia coli K12. J. Antibact. Antifung. Agents, 13, 3-10.

Kourai, H., Takechi, H., Horie, T., Takeichi, K., and Shibasaki, I. (1985b) Quantitative structure-activity relationship of antimicrobial $N$-laurylpyridinium iodides. J. Antibact. Antifung. Agents, 13, 245-253.

Kourai, H., Takechi, H., Kume, M., Takeichi, K., and Shibasaki, I. (1986a) Antimicrobial characteristics of $N, N^{\prime}$-dialkyl- $\gamma, \gamma^{\prime}$-dipyridinium diiodides. $J$. Antibact. Antifung. Agents, 14, 55-63.

Kourai, H., Kume, M., Takeichi, K., Ohtsuru, Y., Ohizumi, M., and Shibasaki, I. (1986b) Antimicrobial characteristics of $\mathrm{N}$-alkyl-5-hydroxyethyl-4methylthiazolium iodides (in Japanese). J. Antibact. Antifung. Agents, 14, 435-442.

Kourai, H., Takechi, H., Muramatsu, K., and Shibasaki, I. (1989) Relationship between hydrophobicity of bacterial cell surface and drug-susceptibility to alkylpyridinium iodides. J. Antibact. Antifung. Agents, 
17, 119-128.

Kourai, H., Hasewgawa, Y., Goto, S., and Nakagawa, K. (1994a) Bacterioclastic action of dodecylpyridinium iodide against Escherichia coli $\mathrm{K} 12$ W3110. J. Antibact. Antifung. Agents, 22. 461-468. Kourai, H., Oda, K., Takechi, H., and Nakagawa, K. (1994b) The antimicrobial characteristics of poly [dimethyliminio (polymethylene) chloride]s. J. Antibact. Antifung. Agents, 22. 519-530.

Kourai, H., Hasegawa, Y., and Wada, K. (1994c) Bactericidal characteristics of $\mathrm{N}$-alkyl-4-butenylpyridinium bromides. J. Antibact. Antifung. Agents, 22, 653-661.

Kourai, H., Manabe, Y., Matsutani, E., Hasegawa, Y., and Nakagawa, K. (1995) Antimicrobial activities of alkylallyldimethylammonium iodides and alkylallyldiethylammonium iodides. J. Antibact. Antifung.
Agents, 23, 271-280.

Krysinski, J. (1990) Rough sets approach to analysis of the structure-activity relationship of quaternary imidazolium compounds. Drug Res., 40, 795-799.

Krysinski, J. (1991) Rough sets approach to analysis of relationship between structure and activity of quaternary pyridinium compounds (in German). Pharmazie, 46, 878-881.

Pavlikova-Moricka, M., Lacko, I., Devinsky, F., Masarova, L., and Mlynarcik, D. (1994) Quantitative relationships between structure and antimicrobial activity of new "soft" bisquaternary ammonium salts. Folia Microbiol., 39, 176-180.

Yabuhara, Y., Kourai, H., and Shibasaki, I. (1972) Antimicrobial activity of $\mathrm{N}$-alkylnicotinamide iodides (in Japanese). J. Ferment. Technol., 50, 86-92. 